FORMATION Formation emploi

Revue française de sciences sociales

102 | avril-juin 2008

Pêle-mêle

\title{
Les « niveaux de formation » à l'heure européenne. Un examen à partir de l'homologation des titres
}

"Vocational training levels" on the European scene. Some ideas about the homologation ofdiplomas

Los "niveles de formación" en Europa. Un examen a partir de la homologación de los títulos

„Ausbildungsniveaus“ innerhalb Europas am Beispiel der Anerkennung von

Titeln

Patrick Veneau et Dominique Maillard

\section{(2) OpenEdition}

Journals

Édition électronique

URL : http://journals.openedition.org/formationemploi/2286

DOI : 10.4000/formationemploi.2286

ISSN : 2107-0946

Éditeur

La Documentation française

Édition imprimée

Date de publication : 1 juin 2008

Pagination : 71-84

ISSN : 0759-6340

Référence électronique

Patrick Veneau et Dominique Maillard, « Les « niveaux de formation » à l'heure européenne. Un examen à partir de l'homologation des titres », Formation emploi [En ligne], 102 | avril-juin 2008, mis en ligne le 01 juin 2010, consulté le 10 décembre 2020. URL : http://journals.openedition.org/ formationemploi/2286 ; DOI : https://doi.org/10.4000/formationemploi.2286

(ㄷ) Tous droits réservés 
Nomenclature

\section{Les « niveaux de formation» à l'heure européenne. Un examen à partir de l'homologation des titres}

Patrick Veneau et Dominique Maillard*

\section{La nomenclature des niveaux de formation issue des travaux de la Planification a forgé à tel point les représentations qu'elles se sont diffusées bien au-delà du système des diplômes du ministère de l'Éducation nationale.}

La construction d'une «Europe de la mobilité et de la connaissance » interroge les cadres nationaux d'éducation et de formation. Ainsi, le processus de Bologne (1999), qui vise à rendre comparables et compatibles les systèmes d'enseignements supérieurs européens (Croché, 2006), instaure, à travers le schéma LMD (licence, master, doctorat), une norme qui ne correspond que partiellement aux cycles universitaires français. Par ailleurs, dans la suite de la « stratégie de Lisbonne » et en lien plus direct avec le Conseil européen de Barcelone de 2004 (Dale, 2006), s'esquisse un «cadre européen des certifications » qui, dans sa forme actuelle, distingue huit niveaux de certifications et d'acquis d'apprentissage (Commission des communautés européennes, 2005 ; 2006). Ces transformations bousculent les repères français institués par la nomenclature des niveaux de formation ( $c f$. encadré 1) et, dans le cas du « cadre européen des certifications » (CEC), instaurent d'autres principes de codification fondés sur les « savoirs", les « aptitudes » et les «compétences ». De ce fait, elles questionnent directement la nomenclature des niveaux de formation et sa pérennité ${ }^{1}$.

${ }^{1}$ L'article 8 du décret relatif à la Commission nationale de la certification professionnelle, qui a succédé, en 2002, à la CTH (Commission technique d'homologation), mentionne que celle-ci est «chargée d'élaborer une nouvelle nomenclature des niveaux de certification, en rapport avec les emplois occupés, et susceptible de permettre des comparaisons européennes et internationales ». Ce n'est que récemment que la Commission nationale de certification professionnelle $(\mathrm{CNCP})$ s'est saisie du problème.

Patrick Veneau et Dominique Maillard sont sociologues au Céreq. Leurs travaux portent sur les politiques de formation. Ils ont publié, entre autres, en 2006 "Les licences professionnelles; formes et sens pluriels de la "professionnalisation" à l'université » dans la revue Sociétés contemporaines, et en 2007: "La formation à l'épreuve de la certification. L'exemple de la Commission technique d'homologation » dans la revue Éducation et Sociétés. 
L'origine de cette nomenclature est bien connue (Tanguy, 2002).

Construite à l'occasion des IVe et Ve plans² (1962/ 1965 et 1966/ à 1970), elle instaure une mise en relation entre la formation et l'emploi. Sa forme la plus aboutie (1969) distingue cinq niveaux de formation et institue - comme le souligne J. Affichard (1983) " une norme des relations entre le système d'enseignement et le système de production». Au cours des décennies 70 et 80 , cette nomenclature et la catégorie de «niveau » sur laquelle elle est construite acquièrent un caractère d'évidence et une autonomie propre à orienter l'expression, le contenu et la gestion des politiques éducatives, notamment en matière d'enseignement professionnel (Tanguy, 1991).

Depuis, certaines transformations - notamment le développement massif de la scolarisation et les phénomènes de "déclassement» mais aussi le succès du discours des «compétences" - sont venues remettre en cause le diplôme comme condition suffisante d'accès à l'emploi et le principe d'équivalence entre formation et qualification ${ }^{3}$. Le développement d'un nouveau référentiel «de marché », observable y compris au sein du secteur éducatif (Éducation et Société, 2005), est-il en passe - à la faveur de la construction du cadre européen d'invalider les instruments mis en place par le référentiel modernisateur de la Planification ? Autrement dit, qu'en est-il aujourd'hui de la force, de la légitimité et in fine de la persistance de cette nomenclature?

Ces interrogations globales peuvent certes faire l'objet d'approches variées; nous nous proposons de les aborder à partir du travail de la Commission technique d'homologation $(\mathrm{CTH})^{4}$. Trois raisons principales justifient l'intérêt de cette instance nationale

\footnotetext{
${ }^{2}$ Mis en place au lendemain de la seconde guerre mondiale, le Plan se veut un instrument de modernisation de l'économie française. Il repose sur la concertation de différentes catégories économiques et sociales sous l'égide de l'État. Les IVe et Ve Plans ont marqué durablement les politiques éducatives.

${ }^{3}$ À des niveaux de formation identifiés et hiérarchisés entre eux correspondent des niveaux d'emploi.

${ }^{4}$ Sur les détails concernant la CTH et l'homologation, le lecteur se référera à l'encadré 3 .
}

pour notre propos. Par sa mission tout d'abord; la commission chargée «d'élaborer une liste de titres et diplômes de l'enseignement technique organisée par niveaux» procède de ce fait à des activités de classement de demandes d'homologation en vue de leur attribuer un niveau. En second lieu, les trente années d'activité de cette commission - créée en 1972, elle fonctionnera jusqu'à 2002 pour être remplacée par l'actuelle $\mathrm{CNCP}$ - la situent précisément dans la période des transformations évoquées. Enfin, en tant qu'instance interministérielle, elle réunit une grande variété des catégories d'acteurs intéressés aux questions de formation : pas moins de dix-sept ministères y sont représentés, les représentants des organisations de salariés et d'employeurs, mais aussi des membres d'organismes intervenant dans la formation professionnelle et des personnalités qualifiées. Du fait de sa position et de sa composition, la commission se révèle un lieu pertinent pour apprécier le caractère unanime ou non du travail de codification et plus généralement pour appréhender l'usage fait de la nomenclature des «niveaux de formation $»$.

L'histoire de la CTH est largement empreinte de l'ensemble des transformations qui ont marqué les politiques de formation et d'emploi. On peut schématiquement distinguer deux grandes périodes. La première permet de souligner comment la commission adhère à l'idée de la formation comme substrat de la qualification (Tanguy, 2004) et participe - par le classement des titres - à réifier les niveaux de formation et à faire apparaître l'équivalence entre formation et qualification comme naturelle et allant de soi (première partie). La seconde période débute en 1993 et s'achève par le remplacement de la CTH par la CNCP. Elle se caractérise par un changement de perspective par lequel la qualification (visée par le titre) et les compétences sont au principe de l'emploi et du classement (deuxième partie). Si les critères changent, le recours à la nomenclature des niveaux de formation reste prégnant et limite de fait l'ambition affichée d'un nouveau mode de classement (troisième partie). L'examen des attributions de classement au «niveau III » viendra exemplifier ces aspects et permettra d'aborder les tensions engendrées par les cadres européens (quatrième partie). 


\section{Encadré 1 \\ Nomenclature \\ des niveaux de formation lapprouvée par décision du Groupe permanent de la formation professionnelle et de la promotion sociale, le 31 mars 1969)}

\section{NIVEAUX I et II}

Personnel occupant des emplois exigeant un niveau de formation supérieur ou équivalent à celui de la licence ou des écoles d'ingénieurs.

\section{NIVEAU III}

Personnel occupant des emplois exigeant normalement une formation du niveau du brevet de technicien supérieur ou du diplôme des Instituts universitaires de technologie, et de fin de premier cycle de l'enseignement supérieur.

\section{NIVEAU IV}

Personnel occupant des emplois de maîtrise ou possédant une qualification d'un niveau équivalent à celui du baccalauréat technique ou de technicien, et du brevet de technicien.

\section{NIVEAU V}

Personnel occupant des emplois exigeant normalement un niveau de formation équivalent à celui du brevet d'études professionnelles (BEP) et du certificat d'aptitude professionnelle (CAP).

\section{NIVEAU $\vee$ bis}

Personnel occupant des emplois supposant une formation courte d'une durée maximum d'un an, conduisant notamment au certificat d'éducation professionnelle ou à toute autre attestation de même nature.

\section{NIVEAU VI}

Personnel occupant des emplois n'exigeant pas de formation allant au-delà de la fin de la scolarité obligatoire.

\section{Encadré2 \\ Méthodologie}

Les observations présentées dans cet article sont issues d'une étude sur l'évolution de l'homologation depuis 1973 (Veneau, Maillard, Sulzer, 2005). Ce travail a été réalisé par le Céreq à la demande de la Direction de l'enseignement scolaire du ministère de l'Éducation nationale (DESCO).

Cette étude s'appuie sur le traitement statistique d'un échantillon de 800 dossiers représentatifs de l'ensemble des dossiers de première demande d'homologation, sur l'exploitation des débats internes à la $\mathrm{CTH}$ (Commission technique d'homologation) larchives internes et comptes-rendus de séances) et l'analyse d'une vingtaine d'entretiens menés auprès d'anciens membres de la $\mathrm{CTH}$, représentatifs de la composition de la commission.

\section{DES PRATIQUES DE CLASSEMENT FAÇONNÉES PAR LES INSTRUMENTS DE LA PLANIFICATION}

Malgré son inscription réglementaire dans une loi sur l'enseignement technologique qui concerne la formation initiale ${ }^{5}$, la CTH est avant tout conçue comme un instrument de promotion de la politique de formation continue, fondée sur la loi de 1971 (Lescure, 2004). Sa principale fonction consiste à attribuer une valeur d'État à des titres dispensés en dehors du ministère de l'Éducation nationale ${ }^{6}$, «quelles que soient les modalités des formations et les formes de contrôles des connaissances retenues $»$.

Le texte de création de la CTH n'ayant pas précisé les modalités concrètes du classement des titres, les réunions préparatoires de la commission sont consacrées à la définition d'un cadre pertinent (nomenclature

${ }^{5}$ Loi $\mathrm{n}^{\circ} 71-577$ du 16 juillet 1971 d'orientation sur l'enseignement technologique (Journal officiel du 17 juillet 1971).

${ }^{6}$ Les titres de ce ministère sont, eux, inscrits de droit. 
et critères). J. Delors, inspirateur de la politique de formation continue envisage, pour sa part, un « système » de classement spécifique à l'homologation, avec "une "grille passe-partout" aux différents niveaux et dans les types de formation [qui constituerait] un système nouveau [n'ayant] pas pour but d'établir des analogies [avec les diplômes]». Cette volonté se comprend en référence au projet d'un système de «formation permanente» qui aurait constitué une alternative à celui de l'École (Delors J., 1974 ; 1976). Néanmoins, l'invention d'un instrument propre à classer les futurs titres homologués et à concrétiser l'idée de contre-modèle au diplôme ne sera pas réalisée.

Les premières réunions montrent que l'appréciation $\mathrm{du}$ « niveau » peut relever de deux ordres de réalité, la formation d'une part, l'emploi d'autre part. La notion de « capacités professionnelles » semble alors s'imposer comme trait d'union entre ces deux termes. C'est ce que doit attester le niveau. Mais très vite, l'examen pratique des demandes ${ }^{7}$ va se focaliser sur la formation, forme objectivée et tangible de l'idée de « capacités professionnelles ».

Dès lors, homologuer un titre - et lui attribuer un «niveau $»^{8}$ - consiste d'abord et avant tout à évaluer une formation, quasi indépendamment de sa valeur d'usage sur le marché du travail et à procéder par comparaison avec les diplômes, définis par des programmes et des contenus, et leur hiérarchie. Cette centration sur la formation imposera l'usage de la nomenclature des niveaux de formation, d'autant plus que cette option est confortée par les recommandations des organismes de production statistique de l'État (Insee, Céreq) présents aux premières réunions de la commission.

Le choix d'une grille construite à partir des diplômes du ministère de l'Éducation nationale et d'un examen

\footnotetext{
${ }^{7}$ Cet aspect de la codification a déjà été souligné (malicieusement) par Desrosières (2001) : "Les “critères" les plus rigoureux sont souvent balayés par l'exploration d'une pile de questionnaires. » Soulignons qu'à l'époque, le contenu des dossiers porte presque exclusivement sur la formation et les moyens matériels et humains de l'organisme.

8 À lire et à analyser les comptes-rendus des débats de la CTH, les actes d'homologation et d'attribution de «niveau » apparaissent très souvent comme deux opérations très imbriquées où le " niveau » découle de manière automatique et immédiate de la décision d'homologation.
}

centré sur la formation tient pour une grande part à la morphologie de l'offre à homologuer. Jusqu'au début des années 80 , près de $90 \%$ des titres à homologuer émanent du ministère du Travail pour des titres dispensés par l'AFPA (Association nationale pour la formation professionnelle des adultes) et, dans une moindre mesure, du ministère de la Défense. En conférant une reconnaissance d'État à ces titres, il s'agit, selon les termes mêmes de la commission, de reconnaître aux individus qui les détiennent une «égale dignité » avec les diplômés de l'Éducation nationale. La nature de l'offre de formation et la prédominance d'un seul registre favorisent l'accord entre les membres de la commission et rendent compte du caractère peu conflictuel associé aux attributions de «niveau », illustré - entre autres - par l'absence de cas de « sous-classement $»^{9}$ de demandes.

En définitive, les deux premières décennies de fonctionnement de la CTH illustrent l'influence des outils issus de la Planification, construits pour établir des relations entre formation et emploi. La formation est au principe de la qualification et la logique de hiérarchisation des diplômes contenue dans la nomenclature des niveaux s'applique également aux titres de la formation continue.

\section{ÉVOLUTION DU CADRE DE RÉFÉRENCE ET NOUVEAUX CRITĖRES POUR L'HOMOLOGATION}

La décennie 80 se caractérise par une intensification et une massification du chômage : l'emploi devient une priorité nationale. Dans ce contexte, la formation va très vite apparaître comme un des principaux leviers de la politique de lutte contre le chômage, mise en œuvre notamment par les gouvernements socialistes successifs. Nombreux sont les exemples de réformes du système d'éducation et de formation qui traduisent cet objectif (Brucy, 2002) : la création des contrats de qualification en 1984 et le développement de l'alternance, la mise en œuvre du baccalauréat professionnel en 1985, la réforme du système

\footnotetext{
${ }^{9}$ Tous les titres sont alors "classés» au niveau demandé par l'organisme de formation.
} 
de financement de la formation professionnelle la même année. Ces mesures ont en commun la volonté de rapprocher les systèmes éducatif et productif (symbolisée notamment par la création, en 1984, du Haut Comité éducation-économie, HCEE) et illustrent la force du présupposé selon lequel la formation peut résoudre le problème du chômage (Sociologie du travail, 1995). Dès lors, la recherche d'une efficacité de la formation s'impose à toutes les composantes du «système » de formation publique, privée, initiale ou continue.

Cette conception de la formation - fortement orientée par des impératifs d'efficacité - a des incidences directes sur la commission d'homologation. $\mathrm{La}$ perspective dans laquelle elle se situait jusqu'alors est questionnée ${ }^{10}$, ce d'autant plus que différentes mesures publiques ont contribué indirectement (par le financement d'un grand nombre d'actions de formation) à la création d'un « marché » de la formation et que la commission est de plus en plus souvent conduite à traiter des demandes émanant d'organismes privés venus y chercher un label. Toutefois, ce n'est que sous l'influence de changements institutionnels profonds que se transformeront sensiblement la physionomie de la commission et ses pratiques. En 1992, les organisations syndicales représentatives sont reconnues comme membres à part entière de la CTH. Si cette dernière demeure une «commission technique» visant strictement à examiner des demandes d'homologation, elle rentre davantage, du fait de sa composition, dans une logique de paritarisme. L'État demeure certes très présent dans son fonctionnement mais la nouvelle composition officialise le rôle qu'il entend octroyer désormais aux « représentants professionnels » - au sens large du terme - en matière de régulation de l'offre de formation professionnelle.

\footnotetext{
${ }^{10} \mathrm{La}$ tenue d'une réunion de la commission permanente du Conseil national de la formation professionnelle, de la promotion sociale et de l'emploi, en mai 1988, cristallise les interrogations et pose les termes d'une perspective alternative à ce qui avait été jusqu'alors l'essence de l'homologation. C'est ce qu'explicite le rapporteur général de la manière suivante: "Il convient aujourd'hui soit de rattacher l'ensemble des titres et diplômes aux "niveaux" du ministère de l'Éducation nationale, qui aurait à trancher sur le point de savoir s'il y a une équivalence ou non, soit de lier l'homologation à la capacité de tenir un emploi déterminé. »"
}

À la même époque, l'IGAS, Inspection générale des affaires sociales, réalise un audit de la commission. Son rapport, aux tonalités très critiques, oppose la pratique routinisée de l'homologation qui se confond avec « un label qualité des formations mises en œuvre pour préparer un titre », à ce qu'elle devrait être, à savoir «un positionnement des titres dans le champ des qualifications et des emplois observables dans les secteurs d'activité ou les entreprises, et un moyen d'améliorer l'évolution de notre appareil de formation aux besoins de l'économie ». Bref, l'homologation doit désormais être résolument au service de l'emploi et s'intégrer dans le cadre plus global de l'action de l'État dans ce domaine. Cet audit se révèle particulièrement important dans l'histoire de la CTH puisqu'il s'accompagne d'un changement de président en avril 1993. Membre de l'IGAS au moment de l'audit, le nouveau président va s'efforcer de situer l'activité de la CTH dans le cadre des préconisations formulées dans le rapport. Il le fera avec une certaine continuité jusqu'à l'arrêt de la CTH en 2002.

Dans le prolongement direct des changements institutionnels, la commission se construit un nouveau cadre de référence, si ce n'est dans la pratique, du moins dans le discours. La notion de « compétence», jusqu'alors très peu utilisée, devient un leitmotiv et avec elle, l'autonomisation des opérations d'évaluation et de validation. Ce faisant, l'ancienne posture, qui consistait à considérer les titres comme des formations, laisse désormais la place à une approche en termes de «certification », résultat d'une évaluation et d'une validation de compétences associées à un objectif professionnel. Ce cadre, dont l'origine est une «instrumentalisation de la formation», aboutit progressivement à dissocier les actions de formation de l'acte de certification (Veneau, Maillard, 2007). Ce faisant, la commission se départit du principe de la Planification selon lequel la formation (définie en termes de durées et de contenus d'enseignement) est à la base de la qualification, qui avait longtemps constitué sa doctrine. Ce changement de doctrine a pu s'opérer de manière d'autant plus rapide que les membres de la commission ont le sentiment de faire œuvre utile, non plus au nom de la promotion sociale mais au nom de l'emploi.

Le changement de perspective de l'homologation conduit la commission à adopter de nouveaux registres 
au premier rang desquels la «qualification visée ${ }^{11} »$. Ce registre acquiert le statut qu'occupait auparavant celui de la formation Ce dernier n'est plus - officiellement - le registre déterminant; il n'en reste pas moins actif dans la pratique de jugement. Si d'autres registres ou critères sont également mobilisés ( « insertion », « pré-requis », « redondance de l'offre ») ils le sont le plus souvent en combinaison avec celui de la qualification. Autrement dit, la nouvelle doctrine de la CTH pourrait être formulée de la manière suivante : «La qualification visée et exprimée en termes de compétence est au principe de l'emploi ».

\section{UNE PERMANENCE DES CLASSEMENTS}

Ce nouveau cadre et les registres qui lui sont associés ont eu un effet limité sur l'attribution des niveaux. Ainsi, sur la période 1993-2000, 90 \% des demandes de titres font l'objet d'un classement au «niveau » demandé par l'organisme de formation Cette observation mérite d'être soulignée car, dans le même temps, la nature des demandes d'homologation s'est largement modifiée. On peut formuler l'hypothèse à ce sujet d'une convergence de représentations entre les « demandeurs » (c'est-à-dire les organismes) pourtant à la recherche d'un effet de label et du « niveau » le plus élevé possible - et les membres de la commission, sur la hiérarchie des « niveaux » et le contenu de chacun d'entre eux.

Examinons plus précisément le traitement d'un échantillon de 120 dossiers dont l'homologation a été décidée au "niveau» demandé ${ }^{12}$. Soulignons d'emblée que dans $80 \%$ de ces cas, il n'est pas question du «niveau » dans les débats : l'expert présente les principales conclusions de son rapport, la commission apporte des compléments de nature administrative et le titre est homologué. Dans le cas des titres envisagés ici, la commission peut fonder

\footnotetext{
${ }^{11}$ Expression utilisée dans le texte.

${ }^{12}$ Les titres du ministère du Travail et ceux du ministère de la Défense ont été volontairement retirés de l'échantillon étudié, dans la mesure où l'homologation était, dans la quasi-totalité des cas, opérée au «niveau » demandé.
}

son jugement sur une conversion non problématique des informations précises contenues dans l'intitulé du titre en niveaux d'emploi. L'ensemble de l'intitulé ou un de ses éléments est suffisamment explicite pour être - par lui-même - classant. Ainsi, le titre «d'agent polyvalent des organismes de tourisme» peut paraître flou en termes d'activité mais le terme « d'agent polyvalent » est associé spontanément à un emploi « d'exécution » identifié au « niveau V ».

Les $20 \%$ des dossiers où il est fait mention du « niveau » ne présentent pas, dans l'ensemble, de différences avec les demandes évoquées ci-dessus. Les « cas » problématiques sont minoritaires ( $f$ f. infra). Pour la majorité des dossiers, les échanges portant sur le «niveau » visent à vérifier le bien-fondé du niveau demandé, quand celui-ci n'est pas spontanément explicité par l'intitulé13. En définitive, c'est moins le niveau que l'intitulé du titre qui donne lieu à discussion.

La codification, procédant de représentations objectivées de la hiérarchie des emplois et des intitulés agissant comme une « cartographie » des emplois, trouve ses limites dans les cas particuliers où l'intitulé contient des informations claires mais contradictoires sur le niveau et empêche l'association spontanée au niveau demandé.

Le titre de «magasinier vendeur» (demandé au « niveau IV ») est, de ce point de vue, exemplaire, l'emploi de magasinier étant situé au «niveau V» quand celui de vendeur l'est au « niveau IV $» \cdot{ }^{14}$ Cette indétermination ne permet pas à la commission de mettre en œuvre sa cartographie globale et classante et la contraint à mobiliser des critères localisés propres au titre présenté (publics, conditions de placement) pour finalement adopter le point de vue de l'organisme demandeur, arguant de l'existence, au travers de cet intitulé, d'une nouvelle qualification.

\footnotetext{
${ }^{13}$ La remarque de la commission suivant laquelle «L'organisme est invité à proposer un intitulé reflétant davantage la qualification et son niveau » (à propos d'un titre intitulé " diplôme d'université qualité » et accepté de manière unanime au «niveau II ») est emblématique de ce cas de figure.

${ }^{14}$ Le titre «d'agent de médiation », évoqué lors des entretiens, en est une autre illustration, le ministère de l'Éducation nationale étant favorable à un classement au «niveau V $\mathrm{V}$ tandis que le ministère du Travail et de l'Emploi plaide pour une attribution au « niveau IV ».
} 
Ci-après l'extrait d'un débat de la commission :

«Au cours du débat, la question du niveau est évoquée par les membres de la commission. [...] Pour conclure, les membres de la commission relèvent les points suivants :

- la qualification de "magasinier vendeur » est bien reconnue au niveau III de la convention collective, niveau correspondant, selon cette même convention, au niveau IV de la nomenclature d'homologation. Ils estiment par conséquent difficile d'homologuer ce titre à un niveau inférieur ;

- la faible reconnaissance du titre sur certains sites semble être liée à une politique d'enseigne (cf. formation en intra au profit de l'entreprise P. ̀̀ Strasbourg).

Le nom de l'organisme responsable de la délivrance du titre est précisé. À l'unanimité, la commission émet un avis favorable à l'homologation du titre au niveau $I V »$.

Ce cas illustre le rôle que jouent les représentations normalisées associant des intitulés d'emploi à un «niveau de qualification». Lorsque cette mise en correspondance implicite s'avère inopérante ou impossible (par exemple quand il s'agit de qualifications nouvelles - le cas le plus notable est celui de la

\section{Encadré 3}

\section{L’homologation des titres}

La CTH (Commission technique d'homologation) a été instituée par la loi de 1971 sur l'enseignement technologique. Elle a été régie par les décrets successifs de 1972, 1977 et 1992 avant de laisser place à la Commission nationale de la certification professionnelle (CNCP) créée le 17 janvier 2002 par la loi de modernisation sociale. La CTH a pour objet « l'inscription sur une liste établie sous l'autorité du Premier ministre des titres et diplômes de l'enseignement technologique, par niveaux, d'une part, par métiers, groupes de métiers ou types de formations, d'autre part ». Au cours de ses trente ans de fonctionnement, la CTH a homologué plus de 5000 titres, dont 30 \% étaient encore " actifs » en 2002, c'està-dire toujours délivrés par un organisme et inscrits sur la liste d'homologation. Sur l'ensemble des titres homologués par la commission, $2 \%$ l'ont été au niveau I de la nomenclature des "niveaux de formation », $9 \%$ au « niveau $\|$ », $22 \%$ au « niveau III », $22 \%$ au « niveau IV » et $45 \%$ au " niveau V ».

La procédure d'homologation repose sur deux étapes successives, la saisine et l'examen. La CTH ne peut être saisie que par une demande transmise soit par le ministère concerné par le titre proposé, soit par le Préfet de Région, président du conseil régional ou recteur dans le cas de demandes émanant d'organismes de formation à compétence régionale. L'instruction proprement dite s'effectue en différentes étapes. L'expert désigné par la CTH présente aux membres de la commission son rapport d'enquête auprès de l'organisme. Par ailleurs, les membres prennent acte de l'avis du COREF (Comité régional de la formation professionnelle, de la promotion sociale et de l'emploi) (dans le cas d'une saisine régionale) et auditionnent les représentants de l'organisme demandeur. Après débat et décision de la commission, le secrétariat transmet un avis (défavorable ou favorable à l'homologation et précisant le niveau et le code de la spécialité du titre) au ministre du Travail qui signe - par délégation du Premier ministre - l'arrêté d'homologation.

Outre le président, le vice-président et le rapporteur général, la commission réunit :

- des représentants de la quasi-totalité des ministères ;

- des représentants des organisations syndicales de salariés et d'employeurs lleur présence a été officialisée par le décret de 19921 ;

- des représentants des chambres consulaires et d'un représentant de la Fédération de l'Éducation nationale ;

- des personnalités qualifiées (par exemple, directeur du CEREQ - Centre d'études et de recherches sur les qualifications, directeur du CNAM - Conservatoire national des arts et métiers, ...). 
qualité qui sera néanmoins tranché assez rapidement - ou d'intitulés trop généraux), les membres de la commission doivent convoquer d'autres registres disponibles, notamment les "pré-requis » exigés à l'entrée en formation (à savoir, l'expérience mais surtout les titres déjà détenus par les candidats) et la formation. Dans ces cas, la formation du jugement peut être influencée par des phénomènes de dynamiques de groupe : "On est sensible à l'argumentation des uns et des autres... Un point est soulevé à la dernière minute... et à ce moment-là, ça fait réfléchir, ça peut faire basculer. » (représentant d'une organisation « intéressée à la formation professionnelle et à la promotion sociale »)

Pour comprendre la quasi-automaticité de ces classements - y compris dans cette seconde période -, il convient de revenir à la relation d'équivalence entre formation et emploi instituée par la nomenclature des «niveaux de formation». Cette nomenclature, utilisée dès les débuts de la CTH pour le classement des formations, a contribué à construire, sur un mode analogique, un espace hiérarchisé des emplois, et c'est précisément cette structure hiérarchisée des emplois qui est mobilisée, après 1993, pour le classement des titres. L'automaticité des attributions de niveaux révèle la naturalisation de l'équivalence. De ce fait, classer des «qualifications visées » ou des «formations» (au cours de la première période) s'apparente, le plus souvent, à des opérations équivalentes, ce qui justifie de classer une « qualification d'agent» comme l'on classerait une «formation d'agent» (i.e. au «niveau V »). De ce fait, il n'y a aucune difficulté à passer d'un registre à l'autre : "Les choses ont été claires: par exemple, une formation d'ouvrier, on savait bien que c'était du niveau V, niveau CAP, et le gars qui avait un peu plus de potentiel pour évoluer on pouvait le situer au niveau IV. Après oui, c'est déjà une fonction d'encadrement, ce n'est pas du cadre, c'est intermédiaire, parfois au niveau III et puis après le cadre qui sait faire, l'expert, tout ça, c'est un cadre niveau I-II. » (représentant d'une organisation syndicale patronale)

On peut ainsi comprendre que la rupture opérée en 1993 ne se soit pas accompagnée d'un changement des modes d'attribution de niveaux, alors même que la commission, dans son ensemble, élabore de nouveaux registres ou critères d'examen des demandes et les instruit selon de nouvelles procédures. S'il en est ainsi, c'est que la nomenclature des niveaux de formation a forgé des représentations partagées et un mode de vision unifié de l'espace professionnel, qui facilite l'accord entre les membres de la commission.

L'attitude même du MEDEF (Mouvement des entreprises de France) est assez symptomatique de cet accord; si cette organisation a défendu l'emploi comme registre principal, elle n'a pas pour autant cherché à introduire des critères spécifiques fondant un système de classement alternatif. En définitive, la commission n'a jamais été pour le CNPF (Centre national du patronat français) puis MEDEF un lieu d'enjeux forts : "J'étais assez favorable à l'homologation parce que l'homologation portait une légitimation supplémentaire qui à mon avis au contraire ne pouvait que conforter la légitimité de la branche qui avait fait son truc. Si la branche fait son truc en essayant de qualifier sa branche et si l'État dit en plus amen c'est parfait. Moi ça me va bien. » (représentant d'une organisation syndicale patronale)

La nomenclature fonctionne ainsi comme schème classant totalement incorporé (Bourdieu, 1986). Qui plus est, son efficacité durable se comprend par sa congruence avec la mise en forme sociale et professionnelle opérée par d'autres nomenclatures, notamment les PCS (catégories socioprofessionnelles) ${ }^{15}$ de l'INSEE. Cette convergence contribue à légitimer les équivalences réalisées.

Toutefois, le principe de la mise en équivalence pendant toute la période n'a pu être assuré qu'en minimisant la valeur des titres sur le marché du travail. Si le registre de «l'insertion» est censé constituer - à partir de 1993 - un aspect déterminant ${ }^{16}$ de l'attribution du niveau, dans la pratique, sa portée restera très modeste. «Je vais vous donner un autre exemple qui, à mon avis, montre la limite de cette évolution de la CTH. Les positionnements se faisaient aussi sur l'analyse du placement des stagiaires. Sauf qu'on n'avait aucune donnée du

\footnotetext{
15 Pour les catégories salariées.

${ }^{16}$ L'audit réalisé en 1992 insistait sur la nécessité de passer à une évaluation des titres a posteriori, c'est-à-dire en intégrant les informations relatives à l'insertion des détenteurs de titres et non plus à une évaluation a priori, i.e. centrée sur la formation.
} 
contexte économique et du marché du travail et que l'ANPE n'était pas membre de la commission. Si vraiment, ce qu'on veut faire c'est voir ce que ça donne en termes de placements, on est obligé de tenir compte de la situation $d u$ marché du travail et de l'évolution économique » (représentant d'un ministère).

De fait, la question du «déclassement» est peu évoquée, y compris pour les diplômes qui servent de repère. En définitive, la codification opérée s'apparente davantage à un idéal-type de classement qu'à une mesure de la valeur des titres sur le marché du travail. En procédant ainsi, la commission assure une pérennité de ses classements, via la nomenclature.

\section{LE « NIVEAU III » DANS LA PERSPECTIVE DU LMD}

Niveau le plus fréquemment attribué à partir de la fin des années 80 , le «niveau III » présente des aspects qui permettent d'illustrer et de prolonger les analyses de la partie précédente. Les pratiques de classement relatives à ce niveau sont l'occasion de montrer un aspect du travail de hiérarchisation opéré spécifiquement par cette nomenclature, à partir du BTS (brevet de technicien supérieur) et du DUT (diplôme universitaire de technologie), les deux diplômes caractéristiques de ce niveau que le schéma du LMD (licencemaster-doctorat) tend à remettre en cause.

\section{Le « technicien supérieur », forme} naturalisée du niveau III de formation

L'augmentation des demandes au niveau III, à partir du milieu des années 80 , et la focalisation de la CTH sur le versant de l'emploi se traduisent, dans les intitulés, par une assimilation de ce niveau à celui de « technicien supérieur $» .{ }^{17}$

Le terme de « technicien supérieur » apparaît pour la première fois, en 1980, dans les débats de la commission, mais il ne se diffuse pas au cours de cette décennie (en 1989, il représente moins de $4 \%$ des intitulés des titres homologués à ce «niveau »). En

\footnotetext{
${ }^{17}$ De la même manière, le terme de «technicien » caractérise les intitulés des titres de niveau IV.
}

revanche, à partir de 1990, il devient de plus en plus fréquent, pour culminer à $57 \%$ après 2000 . Parallèlement à ce développement, il n'est plus cantonné aux emplois de l'industrie mais il est également associé à des activités du commerce, de la gestion.

L'appellation de «technicien supérieur » émerge au milieu des années 50, dans le prolongement de la création des BT (brevets de technicien) en 1952, transformés en 1959 en «brevets de technicien supérieur ». Cette appellation s'inscrit dans un processus visant à clarifier la hiérarchie de l'ensemble des diplômes de techniciens qui - outre ce diplôme le plus élevé - distingue des formations de BEI (brevet d'études industrielles) et de BT (Meylan, 1983). Malgré les tentatives du ministère de l'Éducation nationale pour faire reconnaître sa propre taxonomie (agents techniques, puis techniciens et techniciens supérieurs) dans d'autres espaces, celle-ci n'a jamais été utilisée dans les grilles de classification pas plus que dans la nomenclature des PCS de l'INSEE. Cet intitulé va être repris par la CTH pour substantiver une catégorie d'emplois «de niveau III », à tel point que la commission insistera auprès des organismes pour que cette expression figure dans les intitulés des titres, comme l'illustre le compte-rendu suivant: «Au cours de la délibération, les membres de la CTH proposent à l'unanimité l'homologation de ce titre au niveau III code 201n, mais en retenant un intitulé plus en conformité avec le niveau III : "Technicien supérieur en informatique et électronique industrielles". » (1995)

La « limite supérieure » de cette catégorie n'a pas posé de problème aux membres de la commission qui ont lié - de manière classique - la frontière entre niveau III et niveau II à la question de " l'encadrement ». En revanche, la définition de sa « limite inférieure »-rapportée aux activités ou aux emplois - a été moins évidente. "On partait du fait que le niveau II est cadre et dans l'entreprise le cadre ça a une signification, alors que le technicien supérieur n'est pas cadre [...] Donc, disons que la discussion entre le III et le II, c'était souvent vis-à-vis des centres de formations mais au sein de la commission, je crois qu'il n'y avait pas tellement de problème là-dessus. Par contre, entre le III et le IV oui parce que c'est moins tranché dans le lieu de travail» (entretien 
avec le représentant d'un ministère). Ce qu'atteste l'analyse des titres décrits par les «fonctions » de la nomenclature des spécialités de formation (codes CNIS); les titres de niveaux IV et III se répartissent de manière relativement équivalente, à l'exception des fonctions «d'organisation et de gestion » qui distinguent nettement le «niveau III » et celles liées au « contrôle, prévention, entretien » qui sont davantage représentées au « niveau IV ».

Dans ces conditions, le registre de la formation redevient un " marqueur » de niveau et les demandes sont examinées en référence aux BTS et DUT (diplôme universitaire de technologie), tant sur la question des pré-requis à l'entrée en formation qu'en termes de durées ou de contenus de formation. Il paraît difficile pour la commission de ne pas conférer à un titre un niveau III si ceux qui le préparent sont déjà diplômés $\mathrm{du}$ technique supérieur court. Réciproquement, le niveau III pourrait se trouver " menacé » dès lors que le recrutement des candidats n'est plus réalisé auprès de ces diplômés, comme l'illustre l'exemple suivant : " (Au sujet d'un titre de " Technicien en mécanique sportive »). Après discussion, la commission a trouvé équilibrée cette formation et en adéquation avec ses objectifs et n'a pas jugé utile de proposer des recommandations sauf de maintenir un recrutement au niveau du BTS ou similaire, tel qu'il est pratiqué actuellement. La commission a émis un avis favorable à l'unanimité à l'homologation de ce titre sous l'appellation de "Technicien supérieur en mécanique sportive" " (compte rendu d'une réunion de 1997).

Si le niveau III a été de plus en plus associé à des formations destinées à des candidats déjà diplômés de BTS ou DUT, la commission n'en avait pas moins établi - à force d'examens - une relation entre des titres ouverts à des candidats bacheliers et une durée nécessaire de formation. En-deçà de 1200 heures, le classement à un niveau III s'avérait problématique, au-delà il pouvait être envisagé. Ainsi, dans le cas d'un titre de « Modéliste styliste, industrie du cuir », demandé au niveau IV et accessible à des bacheliers, on peut lire la remarque suivante: "La commission s'étonne que, compte tenu de la durée de la formation, 1800 heures dont 234 en entreprise, le niveau III de sortie ne soit pas demandé. » (compte rendu de réunion, 1994)

Sur la base de ces deux critères (pré-requis à l'entrée et durée de formation), la CTH distinguera pour les activités de commerce et de vente, à la fois un titre de «technicien des forces de vente » au niveau IV (la formation est accessible à des bacheliers ayant une expérience professionnelle d'un an ${ }^{18}$ et dure 1211 heures) et un titre de « technicien supérieur des forces de vente » de niveau III (qui opère un recrutement à bac +2 et s'étend sur une durée de 1318 heures). Le recours à la formation et aux prérequis permettait d'évacuer ainsi le flou relatif des activités et des intitulés qui leur sont associés. À cet égard, le niveau III est à la fois exemplaire de la naturalisation de l'équivalence et typique d'un raisonnement en termes d'emploi.

\section{Le principe de l'équivalence à l'épreuve du LMD}

La commission a catégorisé les bornes du niveau III selon des registres de nature différente : la formation et les «pré-requis» pour la distinction avec le niveau IV, l'emploi (fonction et statut) pour la frontière avec les niveaux II et I spécifiques de la catégorie des cadres. Si ce bornage supérieur peut sembler solidement établi, il n'en a pas moins été interrogé dans les années 90, à la faveur d'un développement de cursus de formation (presque) exclusivement destinés à des diplômés d'IUT (Institut universitaire de technologie) ou de STS (Sections de techniciens supérieurs). Il convient de s'arrêter sur ces demandes qui, à bien des égards, s'apparentent à certaines formations de licences professionnelles créées dans le cadre du LMD, du point de vue des contenus, des durées et des objectifs professionnels poursuivis (Maillard, Veneau, 2006).

C'est en 1985 qu'apparaît pour la première fois une demande d'homologation d'un cursus de ce type. À cette occasion, le vice-président « appelle l'attention des membres de la commission sur le fait que ce type de formation complémentaire [600 heures et deux mois de stage] constitue un précédent $»$. Les demandes, destinées à de jeunes titulaires de l'enseignement supérieur court et organisées le plus souvent sur une durée d'un an, s'accroissent et obligent progressivement la commission à se forger un point

\footnotetext{
${ }^{18}$ Bien qu'évoquée dans ce cas, la mention d'une expérience professionnelle comme substitut au diplôme occupe une place fort modeste, même pour des titres en formation continue.
} 
de vue. Quatre ans plus tard intervient le premier reclassement à un niveau inférieur d'une demande effectuée au niveau II. Les échanges relatifs au classement méritent d'être cités car ils feront jurisprudence :

"Le président s'interroge sur le niveau. Le représentant du ministère de l'Enseignement supérieur estime qu'une $3^{e}$ année de BTS ne correspond pas à un niveau II. Le rapporteur général, quant à lui, souligne que les rémunérations ne semblent pas correspondre à un niveau II, ce que corrobore le vice-président en observant l'évolution professionnelle des anciens stagiaires. En conclusion, la commission propose une homologation au niveau III, groupes 10 et 32, mais souhaite un réexamen du dossier au bout d'un an. Il est demandé à l'organisme de préciser la justification concernant les fonctions et rémunérations des anciens stagiaires. 》 (compte-rendu de réunion, 1991)

On trouve, dans cet extrait, l'essentiel de l'argumentaire selon lequel ces formations doivent être considérées comme des années supplémentaires, des spécialisations de BTS ou de DUT qui, si elles permettent une forme de double compétence ou d'adaptation à l'emploi, ne justifient pas pour autant le saut qualitatif du niveau.

Ce principe sera réinterrogé régulièrement par les organismes de formation désireux d'élever les niveaux de leur offre de formation. La CTH le confirmera néanmoins, comme l'atteste le nombre de déclassements de titres du niveau II au niveau III. Ces cas représentent près des deux tiers de l'ensemble des avis de déclassements. Le critère de l'insertion, associé à celui des contenus - manière détournée d'aborder la question de la durée - vient alors opportunément légitimer le classement adopté. «Après un vote (14 voix pour le niveau III, 1 abstention, 2 voix contre le niveau III), la Commission émet un avis favorable à l'homologation du titre "Diplôme de technologie approfondie en produits financiers » [...] mais au niveau III et sous le libellé "Conseil en produits financiers ». En effet, la Commission relève que le diplôme apparaît essentiellement comme une formation de spécialisation, complémentaire des filières de formation de niveau III. Il ne permet pas de maîtriser les aspects de management, conception et conceptualisation caractéristiques du niveau II. L'homologation prendra effet à compter de 1991. » (compte-rendu des débats, 1997). Comme l'illustre cet exemple, il est courant que les membres de la commission soient amenés à se prononcer non pas pour le «niveau» demandé mais directement pour (ou contre) le niveau III.

Les « reclassements » sont l'occasion d'insister sur le consensus dont fait l'objet ce principe. Très souvent obtenues à l'issue d'un vote, les décisions de déclassements n'en sont pas moins prononcées sans ambiguité. Derrière la défense de cette position s'expriment des arguments communs et des intérêts spécifiques. Peu ou prou, les membres de la commission - au sein de laquelle, rappelons-le, siègent des représentants d'institutions les plus diverses - partagent l'idée que, du point de vue du travail, les activités de techniciens supérieurs se distinguent nettement de celles des cadres et des ingénieurs et que l'accès aux fonctions d'encadrement n'est possible pour de jeunes diplômés qu'au prix d'un titre situé a minima (du point de vue de ses contenus et de sa durée) au niveau du deuxième cycle universitaire ; 1600 heures apparaît ainsi comme la durée minimale requise pour pouvoir prétendre à un titre de niveau II, en dehors de toute prise en compte de l'expérience professionnelle antérieure. Ainsi, «l'année complémentaire» au BTS ou au DUT ne peut être assimilée à une licence. On ne saurait différencier - à travers la position de la commission - ce qui relève d'une analyse en termes d'emploi et de fonctionnement du marché du travail ou d'une interprétation de la nomenclature des niveaux de formation. Par ailleurs, dans le contexte des années 90 marqué par des difficultés d'insertion et un allongement des durées d'études, la politique du ministère de l'Éducation nationale reste dans le cadre de la nomenclature des niveaux. Certes, les formations post DUT ou BTS (sous forme de DU, diplôme d'université, et de FCIL, formation complémentaire d'initiative locale) se développent mais demeurent des formations de niveau III et les IUT n'obtiennent pas gain de cause pour allonger d'un an la durée du DUT ${ }^{19}$. Cette volonté de maintenir au

${ }^{19}$ Même le DNTS (diplôme national de technologie spécialisé), créé à titre expérimental, reste à ce niveau. 
niveau III toute l'offre de l'enseignement supérieur court se comprend d'autant mieux que viennent d'être créés, à des niveaux supérieurs, des diplômes spécifiques (diplôme d'ingénieur-maître des IUP - instituts universitaires professionnalisés, DRT - diplôme de recherche technologique) censés répondre aux besoins techniques des entreprises. Dans ce contexte, il paraissait difficile aux membres de la CTH de brouiller les repères et les équivalences associés à ces niveaux.

À la lumière de ce consensus, on mesure mieux le « coup de force » de $C$. Allègre avec la création de la licence professionnelle dans le projet de construction de l'espace européen d'enseignement supérieur (Charlier, Croché, 2003). En effet, avec la licence professionnelle, l'année supplémentaire octroie de plein droit un niveau supérieur, et ce nouveau diplôme rompt avec l'équation selon laquelle « $2+1$ fait presque toujours III » et que la CTH avait repris à son compte. Ce «niveau » supplémentaire est octroyé sans qu'ait été envisagé l'autre terme de l'équivalence, à savoir le statut (cadre ou non cadre) des emplois auxquels prépare la licence professionnelle. En laissant au seul marché du travail le soin de lever l'indétermination du statut, ce nouveau diplôme altère le principe de mise en correspondance institutionnalisé dans le cadre de la Planification.

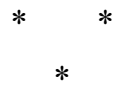

L'analyse des pratiques d'attribution de niveaux par la $\mathrm{CTH}$ montre le caractère structurant de la nomenclature des niveaux de formation issue des travaux de la Planification. Durant les deux premières décennies de fonctionnement, l'homologation est fondée sur une analogie entre diplômes du ministère de l'Éducation nationale et titres à homologuer; elle érige la nomenclature comme mesure de toute chose. Le changement de perspective, opéré en 1993, qui se traduit par une incitation à prendre comme référence les questions d'emploi - appréhendée par le registre principal de la «qualification visée » et le registre secondaire des « débouchés »- aurait pu déstabiliser les repères mobilisés jusqu'alors. Mais le principe d'équivalence, qui fait fonctionner de paire la hiérarchie des qualifications et des emplois et celle des niveaux de formation, a permis une certaine continuité des pratiques de classement.

Si certains auteurs ont pu souligner que "l'équivalence du code est [...] illusoire » (Thévenot, 1992), il n'en demeure pas moins que, dans le cas spécifique de la nomenclature des niveaux de formation, cette « illusion » a montré sa force et son caractère performatif. Les représentations qu'elle a forgées se sont diffusées bien au-delà du système des diplômes du ministère de l'Éducation nationale, au point qu'un raisonnement et un codage en termes de « niveaux d'emploi » ont pu apparaître aux membres de la CTH comme des opérations autonomes, évidentes et indépendantes du caractère normatif de la mise en équivalence des logiques dont elles sont issues.

Dans ces conditions, la construction d'un espace européen de formations et de certifications n'en revêt que plus d'importance dans la mesure où les principes et les normes qu'il tend à imposer se différencient de ceux de la nomenclature des niveaux de formation. Le LMD comme le « cadre européen des certifications » (CEC) viennent perturber les repères propres à la hiérarchie des diplômes et, plus fondamentalement, ils brisent la mise en équivalence essentielle dans le projet de la Planification. Ainsi, le cadre du CEC ne vise plus à réaliser une mise en relation avec le système d'emploi mais à assurer une « transparence (européenne) des certifications » afin, notamment, de "permettre aux employeurs d'apprécier le profil, le contenu et la pertinence des certifications proposées sur le marché de l'emploi $»^{20}$. Le contraste entre cet objectif et la perspective de la mise en équivalence entre niveau de formation et niveau d'emploi, dans laquelle se situait la Planification, est patent.

Si les politiques éducatives sont de plus en plus des «politiques sous influence [européenne]» (Derouet 2006), quel pourra être, dans l'avenir, le rôle des organisations syndicales, patronales et de salariés, sachant que, de longue date, les politiques d'enseignement professionnel et technique se sont construites dans la

\footnotetext{
20 « Proposition de recommandation du parlement européen et du conseil établissant le cadre européen des certifications pour l'apprentissage tout au long de la vie », présentée par la Commission, COM(2006) 479 final, 2006/0163 (COD).

Source : http://www.europarl.europa.eu/oeil/FindByProcnum.do? lang $=1 \&$ procnum $=\mathrm{COD} / 2006 / 0163$
} 
négociation entre ces grandes catégories d'acteurs et l'État. Le compromis social et politique dont procédait la nomenclature des niveaux de formation avait scellé une reconnaissance des diplômes, notamment par l'inscription des principaux diplômes dans les conventions collectives. Les évolutions actuelles lais- sent penser que les bases d'un nouveau compromis devraient s'élaborer au détriment de la mise en équivalence et de la reconnaissance. La « qualité » peutelle constituer un nouvel instrument consensuel pour le pilotage des systèmes d'éducation et de formation (Normand, 2006) ?

\section{Bibliographie}

Affichard J. (1983), « Nomenclatures de formation et pratiques de classement », Formation Emploi, $\mathrm{n}^{\circ} 4$, octobre-décembre, pp. 47-61.

Bourdieu P. (1986), « Habitus, code et codification », Actes de la recherche en sciences sociales, $\mathrm{n}^{\circ} 64$, pp. 40-44.

Brucy G. (2002), « Heurs et malheurs des diplômes professionnels », in Moreau G. (coord.), Les patrons, l'État et la formation des jeunes, La Dispute, pp. 65-78.

Charlier J.-E., Croché S. (2003), « Le processus de Bologne, ses acteurs et leurs complices », Éducation et Sociétés, $\mathrm{n}^{\circ} 12$, pp. 13-33.

Croché S. (2006), "Qui pilote le processus de Bologne? », Éducation et Sociétés, n 18, pp. 203217.

Dale R. (2006), « Construire l’Europe en bâtissant un Espace européen de l'Éducation », Éducation et Sociétés, $\mathrm{n}^{\circ} 18$, pp. 35-54.

Delors J. (1974), «Au-delà des illusions », Esprit, octobre, $n^{\circ} 439$.

Delors J. (1976), «Genèse d'une loi et stratégie de changement », Connexions, ${ }^{\circ} 17$.

Derouet J.-L. (2006), «Entre la récupération des savoirs critiques et la construction des standards du management libéral : bougés, glissements et déplace- ments dans la circulation des savoirs entre recherche, administration et politique en France de 1975 à 2005 », Revue française de pédagogie, $\mathrm{n}^{\circ}$ 154, pp. 5-19.

Desrosières A. (2001), «Entre réalisme métrologique et convention d'équivalence : les ambiguïtés de la sociologie quantitative ", Genèses, $\mathrm{n}^{\circ} 43$, pp. 112-127.

Éducation et Sociétés (2005), La sociologie de l'éducation à l'épreuve des changements sociaux, $\mathrm{n}^{\circ} 16$, numéro spécial, $361 \mathrm{p}$.

Lescure de E. (coord.) (2004), La construction du système français de formation professionnelle continue : retour sur l'accord du 9 juillet 1970 et la loi du 16 juillet 1971, Paris, L'Harmattan, 214 p.

Maillard D., Veneau P. (2006), « Les licences professionnelles ; formes et sens pluriels de la "professionnalisation" à l'université », Sociétés contemporaines, $\mathrm{n}^{\circ} 62$, pp. 49-68.

Meylan F. (1983), « De l'école nationale professionnelle au baccalauréat de technicien ou l'évolution d'une filière de l'enseignement technique ", Formation Emploi, $\mathrm{n}^{\circ}$ 4, octobre-décembre, pp. 29-46.

Normand R. (2006), « De la formation à l'emploi : des politiques à l'épreuve de la qualité », Éducation et Sociétés, nº 18, pp. 5-15.

Sociologie du travail, (1995), La formation contre le chômage?, numéro spécial. 
Tanguy L. (dir.) (1991), Quelle formation pour les ouvriers et les employés en France? La Documentation française, $142 \mathrm{p}$.

Tanguy L. (2002), «La mise en équivalence de la formation avec l'emploi dans les IVe et $\mathrm{V}^{\mathrm{e}}$ Plans (1962-1970) », Revue française de sociologie, 43-4, pp. 685-709.

Tanguy L. (2004), « La fabrication des nomenclatures des niveaux de formation et leur inférence sur la notion de qualification », in Méda D. (dir.), Vennat F. (dir.), Le travail non qualifié : permanences et paradoxes, Paris, Éditions La Découverte, pp. 55-68.
Thévenot L. (1992), « Des chiffres parlants : mesure statistique et jugement ordinaire », in Besson J.-L., La cité des chiffres ou l'illusion des statistiques, Autrement, Série «Sciences en société », pp. 130-142.

Veneau P., Maillard D., Sulzer E. (2005), L'homologation et son évolution depuis 1973, CPC documents, $\mathrm{n}^{\circ} 5,123 \mathrm{p}$.

Veneau P., Maillard D. (2007), «La formation à l'épreuve de la certification. L'exemple de la Commission technique d'homologation », Éducation et Sociétés, pp. 135-148.

\title{
Résumé
}

\section{Les « niveaux de formation » à l'heure européenne. Un examen à partir de l'homologation des titres}

\author{
Par Patrick Veneau et Dominique Maillard
}

Cet article s'inscrit dans le cadre d'une réflexion autour de la nomenclature des " niveaux de formation », interrogée par la logique de la certification et les politiques d'harmonisation européenne. II propose un retour sur les pratiques de classement de la Commission technique d'homologation $(\mathrm{CTH})$, chargée, pendant près de trente ans, d'attribuer un niveau à des titres initialement prévus dans le cadre de la formation continue. II montre que le caractère consensuel des opérations d'attribution de niveau a été rendu possible grâce à une naturalisation des principes et de la logique classificatoire contenus dans cet outil, y compris après le choix de la CTH, en 1993, de privilégier d'autres registres et critères d'examen.

\section{Mots clés}

Niveau de formation, Typologie, Homologation de titre, Formation professionnelle, CTH - Commission technique d'homologation

Journal of Economic Literature : I 21, I 28 